\title{
Rheological Investigation of Self-emulsification Process: Effect of Co-surfactant
}

\author{
Shailesh V. Biradar ${ }^{1}$, Ravindra S. Dhumal ${ }^{1}$, Anant Paradkar ${ }^{1,2}$ \\ ${ }^{1}$ Department of Pharmaceutics, Bharati Vidyapeeth University, Poona College of Pharmacy, Erandwane, Pune 411038 , \\ India; ${ }^{2}$ Institute of Pharmaceutical Innovation, University of Bradford, Bradford, West Yorkshire, BD7IDP, United \\ Kingdom.
}

Received December 02, 2008; Revised March 17, 2009; Accepted June 6, 2009, Published July 22, 2009.

\begin{abstract}
Purpose. The aim of study is to investigate role of co-surfactant in self-emulsification process through rheological analysis of intermediate liquid crystalline (LC) phase formed during selfemulsification. Methods. To mixture of Captex ${ }^{\circledR}$ 200P (C200) and Tween 80 (T80) (SES Plain), either medium hydrocarbon chain co-surfactant $\left(\mathrm{Capmul}^{\circledR} \mathrm{MCM}(\mathrm{CMCM})\right.$ : SES C) or long hydrocarbon chain cosurfactant $\left(\right.$ Peceol $^{\circledR}(\mathrm{P})$ : SES P) was added separately at different concentration levels. Self-emulsification was monitored by visual observations, turbidimetric and droplet size measurement. Mesophases obtained by $30 \% \mathrm{v} / \mathrm{v}$ aqueous hydration of SES were characterized by polarizing microscopy, differential scanning calorimetry (DSC) and rheological studies. Results. SES Plain exhibited 'bad' emulsification owing to instantaneous gel formation in aqueous media. Almost all SES C have shown 'good' emulsification with transparent appearance, very low turbidity value and nano size droplets. All SES P presented 'moderate' emulsification with milky appearance, high turbidity value and coarse droplets. Polarizing microscopy revealed formation of lamellar phase in hydrated SES Plain and in all SES P while almost all hydrated SES $\mathrm{C}$ exhibited formation of micellar cubic phase. In DSC studies, higher extent of LC phase formation was observed in SES C as compared to SES P. Rheological study clearly demonstrated presence of elastic and partially recoverable mesophase in SES Plain, which was transformed into a viscous and non-recovering mesophase with addition of CMCM while there was no change in rheological status of SES Plain after addition of $\mathrm{P}$. The weak and viscous LC phase in SES C must have not presented any resistance to strain induced deformation. Therefore, it might have ruptured easily and quickly, releasing jet of nanosize droplets whereas elastic mesophase in SES P might have ruptured with little resistance resulting in coarse droplets. Conclusion. The ability of co-surfactant to promote self-emulsification was attributed to their influence on viscoelastic properties of intermediate LC phase.
\end{abstract}

\section{INTRODUCTION}

Self-emulsifying system (SES) has emerged as one of the most promising and efficient oral delivery system for lipophilic drugs. Their ability to enhance oral bioavailability of these drugs, ease of manufacturing and commercial applicability present these as delivery system of choice (1-3). SES is isotropic mixture of oil and hydrophilic surfactant, which spontaneously emulsify when introduced in aqueous media under mild agitation. However, a third component, a lipophilic surfactant is added as a co-surfactant to this mixture to decrease emulsification time and droplet size achieved after self-emulsification. The self-emulsification is observed with particular co-surfactant and specific ratio of oil: surfactant: co-surfactant can lead to efficient self-emulsification i.e. emulsification with lower or nano sized droplets.
However, the ability of co-surfactant with a specific composition to promote selfemulsification is still not completely revealed $(4,5)$.

In spite of various attempts to understand the mechanism of self-emulsification, the process is still not well elucidated $(3,5)$. However, according to Reiss (6), self-emulsification occurs when the entropy change that favours dispersion is greater than the energy required to increase the surface area of the dispersion. For a system to self-emulsify, the interfacial tension between oil and water interface must be very low or negative and hydrophilic surfactant alone or in combination with lipophilic co-surfactant lowers the interfacial tension to a very low value (7).

Correspondence Author: Prof. Anant R. Paradkar, Institute of Pharmaceutical Innovation, University of Bradford, Bradford, West Yorkshire, BD7 IDP, United Kingdom.

E-mail: A. Paradkar@Bradford.ac.uk 
ABBREVIATIONS. CIPAC, Collaborative International Pesticide Analytical Council; CMCM, Capmul $^{\circledR}$ MCM; C200, Captex ${ }^{\circledR}$ 200P; DSC, differential scanning calorimetry; DW, distilled water; $D_{50}$, volume median diameter; $G$, elastic modulus; G', loss modulus; Hz, Hertz; LC, liquid crystalline; LVR, linear viscoelastic region; $\mathrm{P}$, Peceol; Pa, Pascal; SBW, structurally bound water ratio; SEDDS, self-emulsifying drug delivery system; SES, self-emulsifying systems; SES C, SES with Capmul ${ }^{\circledR}$ MCM as co-surfactant; SES P, SES with Peceol ${ }^{\circledR}$ as co-surfactant; SD, standard deviation; T80, tween 80 ; $\tan \delta$, loss tangent; $\delta$, phase degree

Improvement in self-emulsification with addition of co-surfactant was attributed to their ability to increase the fluidity of interfacial surfactant film and thereby decreasing bending stress at droplet curvature, which results in smaller droplets after emulsification $(7,8)$. Many researchers have claimed that self-emulsification proceeds through formation of LC phase at interface and the rate and extent of water penetration into LC phase determines the rate of emulsification (9-13). Although, formation of LC phase during selfemulsification was proved both theoretically and practically, correlation between spontaneous emulsification and LC phase formation is still not fully established (4).

It was noted that efficient selfemulsification is observed with specific cosurfactant and with particular ratio of oil: surfactant: co-surfactant. As self-emulsification is very rapid and spontaneous process, it is not possible to monitor it with normal set of experimental conditions. Therefore, arresting this process at a critical stage will be more advantageous to investigate self-emulsification ability of specific SES composition. Previous reports proved that self-emulsification proceed through LC phase formation and its subsequent rupture due to water penetration, but the effect of physical and structural properties of LC phase on self-emulsification performance has not been thoroughly explored. Rheological measurement is proved as a sensitive tool to investigate the microstructure of a system. Therefore, it will be noteworthy to investigate the effect of viscoelastic properties of intermediate LC phase obtained by controlled hydration of SES on selfemulsification performance. In our previous study, we have proved the role of viscoelastic properties of intermediate LC phase in selfemulsification process of oil: surfactant mixture in absence of co-surfactant by using rheological tools (14). It was concluded that droplet size achieved after self-emulsification is function of nature and pattern of LC phase rupture, which in turn depend on viscoelastic properties of formed LC phase. With this background, the role of cosurfactant in self-emulsification can also be revealed through rheological analysis of intermediate LC phase. Hence, aim of the present work is to study the effect of co-surfactant and its hydrocarbon chain length on self-emulsification process through rheological analysis of intermediate LC phase.

\section{MATERIALS AND METHODS}

Materials. Captex ${ }^{\circledR}$ 200P (C200: mixed diesters of caprylic/capric acid of propylene glycol) and Capmul $^{\circledR}$ MCM (CMCM: Glyceryl mono- and dicaprate: HLB: 5-6) were generous gift samples from Abitec Corporation, USA. C200 has the following fatty acid distribution, Caprylic $\left(\mathrm{C}_{8}\right)$ : $77 \%$ and Capric $\left(\mathrm{C}_{10}\right): 21.7 \%$. $\mathrm{CMCM}$ is approximately $1: 1$ mixture of $\mathrm{C}_{8} / \mathrm{C}_{10}$ mono/diglycerides with $2 \%$ free glycerol and has following fatty acid composition, Caprylic $\left(\mathrm{C}_{8}\right)$ : $84.3 \%$ and Capric $\left(\mathrm{C}_{10}\right): 15.7 \%$. Peceol ${ }^{\circledR}(\mathrm{P}$ : Glyceryl monooleate: HLB: 3-4) was gift sample from Gattefosse, France. It has $44 \%$ monoglycerides, $44 \%$ diglycerides and $10 \%$ triglycerides. The fatty acid composition of $\mathrm{P}$ is oleic acid $\left(\mathrm{C}_{18: 1}\right): 76.6 \%$ and linolenic acid $\left(\mathrm{C}_{18: 2}\right)$ : $11.7 \%$. Tween 80 (T80: Polyoxyethylene 20 sorbitan monooloeate) was purchased from Merck Chemicals, Mumbai, India. Distilled water was used for hydration of SES.

Formulation of SES. All lipid excipients were warmed at $45^{\circ} \mathrm{C}$ for $1 \mathrm{~h}$ before mixing on a controlled temperature water bath. C200 and T80 were mixed homogenously at 1:1 ratio (SES Plain) and then CMCM or P was added separately to this mixture at five different concentration levels viz., 20, 40, 60, 80 and $100 \% \mathrm{w} / \mathrm{w}$ of oil: surfactant mixture. SES with CMCM were termed as SES C while those prepared with $\mathrm{P}$ were denoted as SES P (Table 1). Prepared SES (unhydrated) were used for visual observation, turbidimetric and droplet size measurement.

Hydration of SES. The prepared SES were hydrated at 10, 30, 50 and $70 \% \mathrm{v} / \mathrm{v}$ hydration levels with distilled water and stored for $24 \mathrm{~h}$. SES with optimum hydration were used for further evaluations. 


\section{Evaluation of SES}

\section{Emulsifying properties}

Emulsifying properties of SES were visually observed as per Collaborative International Pesticide Analytical Council (CIPAC) test (15). A fixed amount $(0.6 \mathrm{~mL})$ of SES were added to 400 $\mathrm{mL}$ distilled water at $25^{\circ} \mathrm{C}$ and performance of spontaneous emulsification was observed visually and analyzed according to droplet formation pattern. The appearance of formed emulsion was also considered for evaluation of self-emulsifying properties of SES. This test was performed in triplicate.

\section{Droplet size measurement}

Droplet size was measured by Laser Diffractometer (LD) Mastersizer 2000 Ver. 2.00 (Malvern Instruments, Malvern, UK). The data was presented in terms of volume median diameter $\left(\mathrm{D}_{50}\right)$ and span, which were calculated by Malvern Software Ver 3.0. For measurement, SES were pre-diluted by addition of $0.6 \mathrm{~mL}$ of SES to $90 \mathrm{~mL}$ of distilled water under slow agitation at room temperature $\left(25^{\circ} \mathrm{C}\right)$. The system was analyzed by dispersing it in $100 \mathrm{~mL}$ distilled water as dispersant. Analysis was done in triplicate and mean results are presented.

\section{Turbidimetric evaluation}

Nepheloturbidimetric evaluation was done to monitor the progress of emulsification. SES (0.1 $\mathrm{mL})$ was added to $0.1 \mathrm{~N}$ hydrochloric acid (150 $\mathrm{mL})$ under continuous stirring $(10 \mathrm{rpm})$ on magnetic plate (Ika-Werke, Staufen, Germany) at $25^{\circ} \mathrm{C}$. The increase in turbidity was measured using a turbidimeter (Type 131, Systronics, Ahmedabad, India). However, since the time required for complete emulsification was too short, it was not possible to monitor the rate of change of turbidity (rate of emulsification). All observations were carried out within 0-1000 NTU range. Analysis was done in triplicate.

\section{Polarizing light microscopy}

Hydrated SES were transferred to a specially fabricated glass tube (internal diameter $0.5 \mathrm{~cm}$ ) and then viewed for presence or absence of birefringence under polarizing microscope at $25^{\circ} \mathrm{C}$ with $\lambda 1 / 4$ compensator (Nikkon Eclipse $\mathrm{E}$ 600 , Nikon Instech Co., Japan) and under 40X magnification.

\section{Differential scanning calorimetry (DSC)}

DSC measurements were performed with Mettler Toledo 821e instruments equipped with an intracooler (Mettler Toledo, Switzerland). A $10 \pm$ $3 \mathrm{mg}$ of hydrated SES was placed in closed aluminum crucibles and subjected to heating from -20 to $100{ }^{\circ} \mathrm{C}$ and the scanning rate was $5{ }^{\circ} \mathrm{C} / \mathrm{min}$. Calibration was performed with Indium/zinc standards.

\section{Rheological studies}

Rheological measurements of hydrated SES were performed using a controlled stress rheometer (Viscotech Rheometer, Rheologica Instruments AB, Lund, Sweden). Data analysis was done with Stress RheoLogic Basic software, version 5.0. A cone and plate geometry was used with $25 \mathrm{~mm}$ diameter and cone of $1.0^{\circ}$. Fresh sample was used for every test and all measurements were carried out at $25^{\circ} \mathrm{C}$. All tests were carried out in triplicate.

Viscometry: The samples were exposed to increasing stress $(1-50 \mathrm{~Pa})$ and relation between shear stress and shear rate was studied.

Oscillation stress sweep: Linear viscoelastic region (LVR) was determined in the stress range of 1-50 Pa and at a constant frequency $(0.25 \mathrm{~Hz})$. The ability of hydrated SES to resist the deformation with applied stress was recorded in terms of trends of elastic modulus (G') and loss modulus (G').

Oscillation frequency sweep: The samples were exposed to increasing frequency $(0.01-1.0 \mathrm{~Hz})$ at a constant stress within LVR. Effect of frequency on $\mathrm{G}^{\prime}, \mathrm{G}^{\prime \prime}$, phase degree $(\delta)$ and loss tangent (tan $\delta)$ were recorded.

Creep-recovery: SES samples were exposed to a constant stress in LVR for 100s. It was then instantly removed and the recovery was followed for 200s. The creep compliance, J (defined as ratio between measured strain and applied stress) was recorded against time. 


\section{RESULTS}

\section{Emulsifying properties}

Visual test applied in this study was modification of CIPAC test (15). The tendency of spontaneous emulsification and appearance of formed emulsion were taken in consideration. 'Good' emulsification was noted when droplets are formed spontaneously in water resulting in a transparent emulsion while it was noted as 'moderate' for spontaneous emulsification with milky appearance (Table 1).

In case of SES Plain, instantaneous formation of turbid gel was observed on addition to water. Therefore, this mixture without cosurfactant was considered as 'bad' emulsifying system as spontaneous emulsification was not observed. Emulsifying properties of SES C with lower concentration of CMCM (SES 20\%, 40\% and $60 \%$ C) was considered as 'good' as spontaneous emulsification with formation of transparent emulsion was observed for these SES. SES $80 \% \mathrm{C}$ and SES $100 \% \mathrm{C}$ have shown spontaneous emulsification with slight milky appearance; therefore have 'moderate' emulsifying properties. All SES $P$ have 'moderate' emulsifying properties and have produced milky emulsion. The observed difference between SES $80 \%$ and $100 \% \mathrm{C}$ and all SES $P$ was presence of large dispersed oil droplets after emulsification in later case.

\section{Droplet size measurement}

Droplet size measurement of all studied SES is shown in Table 1. Droplet size measurement of SES Plain was not feasible due to formation of gel in aqueous media. Observed volume median diameter for almost all SES C was in nano size except in SES $100 \%$ C, where observed droplet size was close to one micron. It was further noted that as CMCM concentration was increased, there was an increase in droplet size. However, such correlation was missing in SES P. For all SES P, droplets observed after emulsification were in micron size. Among all SES C, SES 100\% C has presented larger droplet size after emulsification. However, in case of all SES P, the observed droplet size was much larger than that of SES $100 \% \mathrm{C}$.

\section{Turbidimetric evaluation}

In the turbidity measurement, the amount of scattered light is measured and used in turbidity calculations as per the Rayleigh's theory (16). The turbidity of the solution is function of the size of droplets, number of droplets and the refractive index difference between medium and the droplets (17). Turbidity measurement of SES Plain was not feasible due to gel formation. From Table 1, it was concluded that turbidity value of SES P was higher than SES C. Secondly, for SES $\mathrm{C}$, it was observed that turbidity increased with an increase in concentration of CMCM while such co-relation was missing in SES P.

\section{Polarizing light microscopy}

The characterization of the LC phase formed after hydration of SES was done under polarizing light microscope and based on Rosevear (18) and Ivanova et al. (19) classification system. Prepared SES were hydrated at 10, 30, 50 and $70 \% \mathrm{v} / \mathrm{v}$ with distilled water and analyzed for phase characterization. The $30 \% \mathrm{v} / \mathrm{v}$ hydration level was selected as LC phase were not formed at $10 \%$ hydration level while at higher hydration level, emulsification was observed. Secondly, higher hydration level produced very low viscous system especially in case of SES 80\% P and SES 100\% $\mathrm{P}$, which presented practical difficulties in use of common rheological geometry for both SES C and SES P. For all further measurements, SES were hydrated at $30 \% \mathrm{v} / \mathrm{v}$ with distilled water.

The observed LC phases in the hydrated SES are shown in Figure 1 and Table 2. In case of SES Plain, birefringence was observed. Among SES C, only SES $20 \%$ C presented birefringence while all other SES C have shown dark texture. The SES 20\% C with comparatively lower concentration of $\mathrm{CMCM}$ must be in transition mode where complete transformation of lamellar phase must have not processed owing to low CMCM concentration. All SES $\mathrm{P}$ exhibited birefringence under polarizing microscope (Table 2). Thus, lamellar phase formation was observed in SES Plain, SES 20\% C and all SES P while micellar cubic phase was present in rest of the studied SES C. (20-22). 
Table 1. Composition, droplet size, turbidity and emulsification properties of various SES

\begin{tabular}{|c|c|c|c|c|c|c|}
\hline \multirow{2}{*}{$\begin{array}{c}\text { Self- } \\
\text { emulsifying } \\
\text { system }\end{array}$} & \multicolumn{2}{|c|}{$\begin{array}{c}\text { Concentration } \\
\text { of co- } \\
\text { surfactant } \\
(w / w) \\
\end{array}$} & \multicolumn{2}{|c|}{$\begin{array}{l}\text { Droplet size } \\
\text { measurement }\end{array}$} & \multirow[t]{2}{*}{$\begin{array}{l}\text { Turbidity }^{\mathrm{a}} \\
\text { NTU }\end{array}$} & \multirow[t]{2}{*}{ Emulsification properties ${ }^{c}$} \\
\hline & $\begin{array}{c}\text { CMC } \\
\text { M }\end{array}$ & $\mathbf{P}$ & $\left(D_{50}\right) \mu m^{a}$ & $\operatorname{Span}^{\text {a, b }}$ & & \\
\hline SES Plain & NA & NA & NA & NA & gelling & $\begin{array}{l}\text { 'Bad' emulsification with } \\
\text { gelling }\end{array}$ \\
\hline SES $20 \% \mathrm{C}$ & $20 \%$ & NA & $\begin{array}{l}0.101 \pm \\
0.00058\end{array}$ & $\begin{array}{l}0.820 \pm \\
0.00336\end{array}$ & $11 \pm 0.283$ & $\begin{array}{l}\text { 'Good' transparent } \\
\text { nanoemulsification }\end{array}$ \\
\hline SES $40 \% \mathrm{C}$ & $40 \%$ & NA & $\begin{array}{l}0.102 \pm \\
0.00048\end{array}$ & $\begin{array}{l}0.819 \pm \\
0.00469\end{array}$ & $2 \pm 0.063$ & $\begin{array}{l}\text { 'Good' transparent } \\
\text { nanoemulsification }\end{array}$ \\
\hline SES $60 \% \mathrm{C}$ & $60 \%$ & NA & $\begin{array}{l}0.104 \pm \\
0.00039\end{array}$ & $\begin{array}{l}0.951 \pm \\
0.00269\end{array}$ & $5 \pm 0.126$ & $\begin{array}{l}\text { 'Good' transparent } \\
\text { nanoemulsification }\end{array}$ \\
\hline SES $80 \% \mathrm{C}$ & $80 \%$ & NA & $\begin{array}{l}0.961 \pm \\
0.00688\end{array}$ & $\begin{array}{l}3.07 \pm \\
0.018810\end{array}$ & $100 \pm 2.21$ & $\begin{array}{l}\text { 'Moderate' turbid } \\
\text { microemulsification }\end{array}$ \\
\hline SES $100 \% \mathrm{C}$ & $100 \%$ & NA & $\begin{array}{l}1.258 \pm \\
0.01021\end{array}$ & $\begin{array}{l}3.30 \pm \\
0.023430\end{array}$ & $138 \pm 4.22$ & $\begin{array}{l}\text { 'Moderate' turbid } \\
\text { microemulsification }\end{array}$ \\
\hline SES $20 \% \mathrm{P}$ & NA & $20 \%$ & $\begin{array}{l}3.56 \pm \\
0.06336\end{array}$ & $\begin{array}{l}3.90 \pm \\
0.04875\end{array}$ & $217 \pm 7.42$ & $\begin{array}{l}\text { 'Moderate' turbid } \\
\text { microemulsification with large } \\
\text { oil droplet }\end{array}$ \\
\hline SES $40 \% \mathrm{P}$ & NA & $40 \%$ & $\begin{array}{l}16.22 \pm \\
0.31304\end{array}$ & $\begin{array}{l}2.513 \pm \\
0.03945\end{array}$ & $170 \pm 4.54$ & $\begin{array}{l}\text { 'Moderate' turbid } \\
\text { microemulsification with large } \\
\text { oil droplet }\end{array}$ \\
\hline SES $60 \% \mathrm{P}$ & NA & $60 \%$ & $\begin{array}{l}11.906 \pm \\
0.24157\end{array}$ & $\begin{array}{l}2.934 \pm \\
0.05515\end{array}$ & $102 \pm 2.14$ & $\begin{array}{l}\text { 'Moderate' turbid } \\
\text { microemulsification with large } \\
\text { oil droplet }\end{array}$ \\
\hline SES $80 \% \mathrm{P}$ & NA & $80 \%$ & $\begin{array}{l}11.380 \pm \\
0.16842\end{array}$ & $\begin{array}{l}6.143 \pm \\
0.07248\end{array}$ & $169 \pm 3.55$ & $\begin{array}{l}\text { 'Moderate' turbid } \\
\text { microemulsification with large } \\
\text { oil droplet }\end{array}$ \\
\hline SES $100 \% \mathrm{P}$ & NA & $100 \%$ & $\begin{array}{l}5.213 \pm \\
0.06047\end{array}$ & $\begin{array}{l}6.882 \pm \\
0.09428\end{array}$ & $\begin{array}{l}426 \pm \\
13.54\end{array}$ & $\begin{array}{l}\text { 'Moderate' turbid micro- } \\
\text { emulsification with large oil } \\
\text { droplet }\end{array}$ \\
\hline
\end{tabular}

A, mean \pm S.D. $(n=3)$; b, Span= d(0.9)-d(0.1)/d(0.5); c, performed in triplicate

\section{Differential scanning calorimetry (DSC)}

The formation of LC phase in SES results from interaction of water molecule with the surfactant. Gati et al. (23) reported presence of bound and free water in surfactant based systems with LC phase formation. Bound water, which is consumed for the formation of LC phase, has different thermal properties as compared to free or unbound water. It is reported that the state of water in surfactant based system is indicated by position of the peak in DSC thermal profile.

During DSC studies of such system, peak corresponding to melting of unbound water appears close to $0^{\circ} \mathrm{C}$ (24). Thermal profiles of SES were studied for presence of an endothermic peak corresponding to free water close to $0{ }^{\circ} \mathrm{C}$. Thermal profile of SES Plain, distilled water (DW) and different SES C are given in Figure 2. 


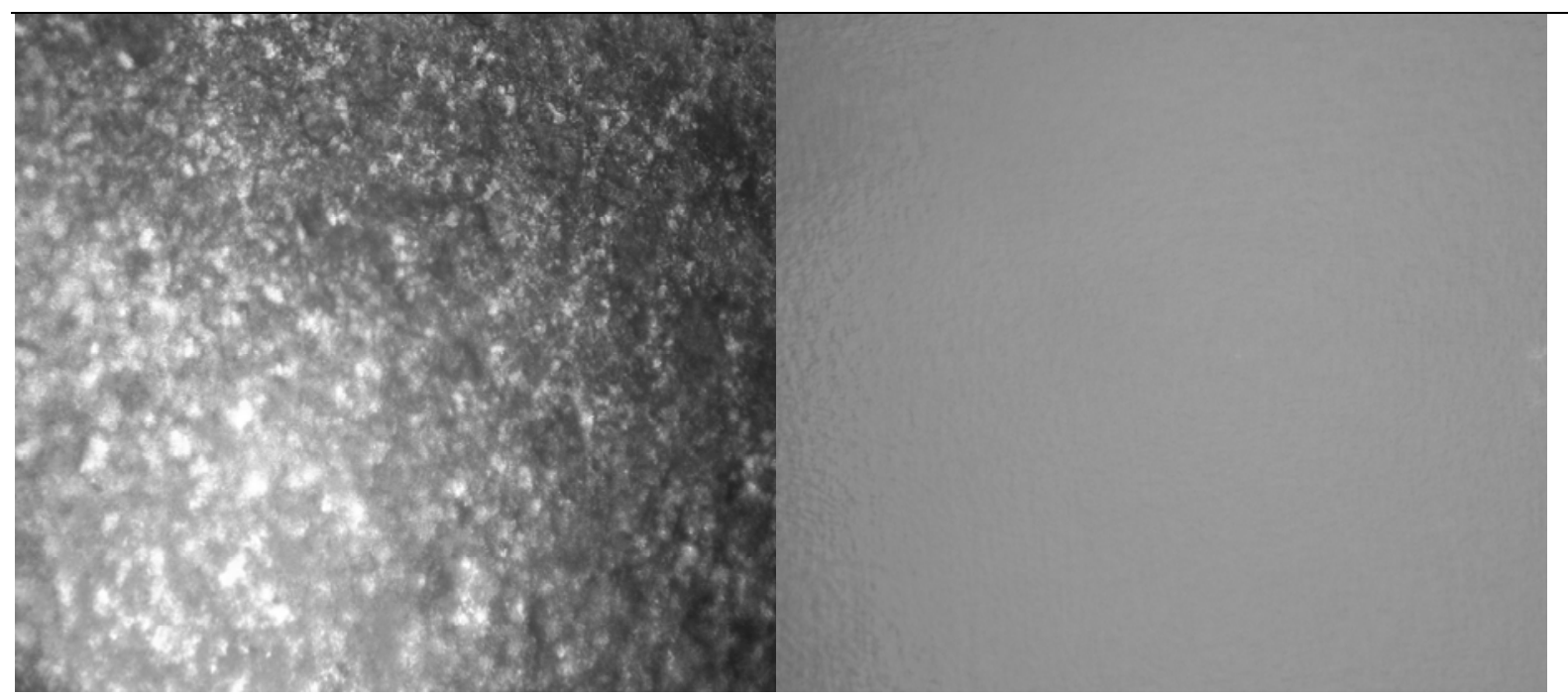

A

B

Figure 1. Photographs of different LC phase observed under polarizing light microscope in studied SES: A- Lamellar phase; B- Micellar cubic phase.

Table 2. Phase characterization of different SES using plain polarizing microscopy

\begin{tabular}{ccll}
\hline Sr. No. & SES & Texture analysis & Phase identified \\
\hline 1 & SES Plain & birefringence & lamellar \\
2 & SES $20 \% \mathrm{C}$ & birefringence & lamellar \\
3 & SES $40 \% \mathrm{C}$ & dark background & micellar cubic \\
4 & SES $60 \% \mathrm{C}$ & dark background & micellar cubic \\
5 & SES $80 \% \mathrm{C}$ & dark background & micellar cubic \\
6 & SES $100 \% \mathrm{C}$ & dark background & micellar cubic \\
7 & SES $20 \% \mathrm{P}$ & birefringence & lamellar \\
8 & SES $40 \% \mathrm{P}$ & birefringence & lamellar \\
9 & SES $60 \% \mathrm{P}$ & birefringence & lamellar \\
10 & SES $80 \% \mathrm{P}$ & birefringence & lamellar \\
11 & SES $100 \% \mathrm{P}$ & birefringence & lamellar \\
\hline
\end{tabular}

For SES Plain, there was no endothermic peak close to $0{ }^{\circ} \mathrm{C}$ corresponding to the melting of free water, similar to that for pure water (Figure 2: DW). This endothermic peak of free water was seen only in thermal profile of SES $40 \% \mathrm{C}$.

Thus, it was concluded that free water was completely utilized for formation of LC phase in
SES Plain and all SES C except SES $40 \%$ C. In case of SES P, SES 20\% P thermal profile had not shown any endothermic peak for free water. All other SES P have exhibited an endothermic peak for free water, thus confirming partial utilization of added water for formation LC phase (Figure 3). In SES $20 \% \mathrm{P}$, due to comparatively high 
hydrophilic surfactant concentration, added water was completely utilized for formation of LC phase.

\section{Rheological studies}

Rheological analysis was performed to investigate viscoelastic properties of LC phases formed after $30 \% \mathrm{v} / \mathrm{v}$ hydration of SES. Measurements were performed by steady- state flow (rotational) and dynamic (oscillatory) measurement modes. The rheological data of SES Plain and SES 20\%, 40\% and $60 \%$ from SES C and SES P is reported as SES with higher concentration of co-surfactant followed the same trend as that of SES $60 \%$ of respective SES class.

The shear rate and shear stress profiles of the SES Plain, SES $40 \% \mathrm{C}$ and SES $40 \% \mathrm{P}$ are shown in Figure 4. SES Plain and SES 40\% $\mathrm{P}$ have shown non-Newtonian relationship (pseudo plastic) between shear stress and shear rate while direct relationship was seen in SES 40\% C. Therefore, formation of lamellar LC phase was confirmed in SES Plain and all SES P, where as formation of micellar cubic phase was exhibited by almost all SES C. SES $20 \%$ C exceptionally exhibited non-Newtonian relationship owing to lamellar phase formation (25).

Hydrated SES were subjected to an increasing stress and trends of G' were observed to calculate LVR (Table 3). For SES Plain, G' was much higher than $G$ ', throughout the applied stress range and also has high LVR, which indicated presence of a strong and elastic gel structure (26). With addition of CMCM as cosurfactant to SES Plain, G' was tremendously reduced and situation was reversed i.e. G" was greater than G' over entire stress. Same trend was observed in all SES C. These findings suggested presence of weak and viscous intermediate LC phase structure in SES C. Opposite trend of G' and G" was observed when CMCM was replaced by $\mathrm{P}$ as a co-surfactant. The elastic nature of SES Plain was maintained in SES $\mathrm{P}$ within LVR region although elasticity was slightly decreased with addition of $P$. The observed LVR for both SES C and SES $P$ was narrow than that of SES Plain, indicating reduction in gel strength. As LVR for SES C and SES P was totally different; the different stress were selected for subsequent frequency sweep (27).

For all SES C, frequency sweep was carried out at a stress of $20 \mathrm{~Pa}$ and for all SES P; stress was fixed at $1.5 \mathrm{~Pa}$. For comparison, frequency sweep of SES Plain was carried out both at $20 \mathrm{~Pa}$ (SES Plain I) and 1.5 Pa (SES Plain II). Similar trends of G' and G' over applied frequency were observed for both SES Plain I \& II, even though the stress was varied. In both cases, $G^{\prime}$ was much greater than G' and both were stable over applied frequency range (Figure 5).

In SES C, the trend of G' and G' was reversed as compared to SES Plain (Figure 6). The G" was monotonously increasing over the applied frequency and was much greater than G' over entire frequency. Exactly opposite trend was observed in SES P where G' was greater than G', over applied frequency (Figure 7).

In stress sweep, SES $P$ have presented elastic behavior within LVR and viscous behavior above LVR whereas in frequency sweep, elastic behavior was observed over entire frequency range. This observed discrepancy may be due to the ability of frequency sweep to represent the true rheological ground state of the material as it was performed within LVR (26).

Based on observed phase degree values, rheological behavior of a system can be predicted (28). The elastic nature of LC phase in SES Plain was confirmed by its $\delta$, which was less than $45^{\circ}$ and shifted towards highly elastic state with applied frequency (Figure $8 \& 9$ at $20 \mathrm{~Pa}$ and 1.5 $\mathrm{Pa}$, respectively). The $\delta$ values for SES C were in viscous mode (close to $90^{\circ}$ ) (Figure 8 ), while in SES P; $\delta$ was less than $45^{\circ}$ (Figure 9). Therefore, elastic behavior was seen in SES Plain and SES P while viscous behavior was seen in SES C.

Loss tangent is ratio of G' to G' and indicate rheological nature of system. The division of material as elastic or viscous is based on observed value of $\tan \delta$ (27). For SES C, it was well above one while in SES Plain and SES P, it was less than one (Figure $10 \& 11$, respectively). Thus, intermediate elastic LC phase was seen in SES Plain and SES $\mathrm{P}$ while viscous LC phase was observed in SES C (27).

In creep recovery, the viscoelastic property of material is determined from recovery component in terms of instantaneous elastic recovery and elastic recovery (29). LC phase in SES Plain has shown complete instantaneous elastic recovery (Figure 12: Region A-B) and partial elastic recovery (Figure 12: Region B-C) after removal of stress. Trend was similar for SES plain at both 20 and $1.5 \mathrm{~Pa}$. Exactly opposite trend of creep recovery was observed for SES C and SES P (Figure $13 \& 14$, respectively). In SES C, neither instantaneous elastic nor elastic recoveries were present while in SES P both were present. 


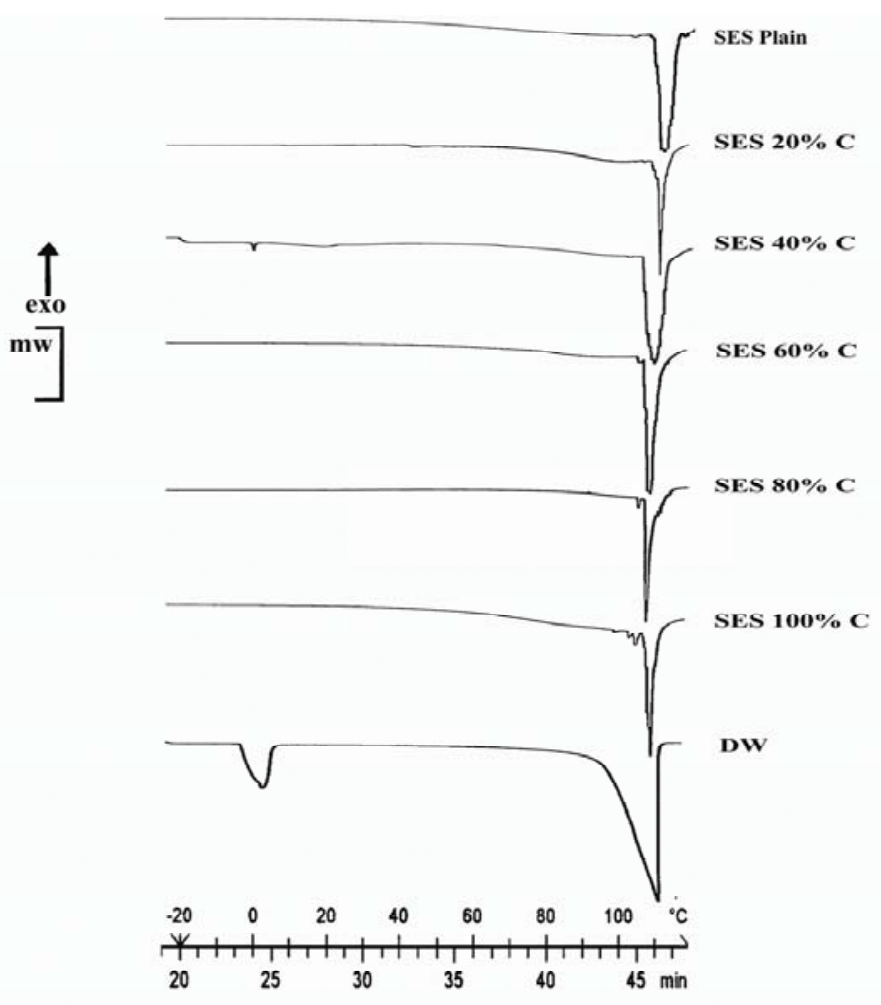

Figure 2. Thermal profiles of SES Plain, distilled water (DW) and different SES

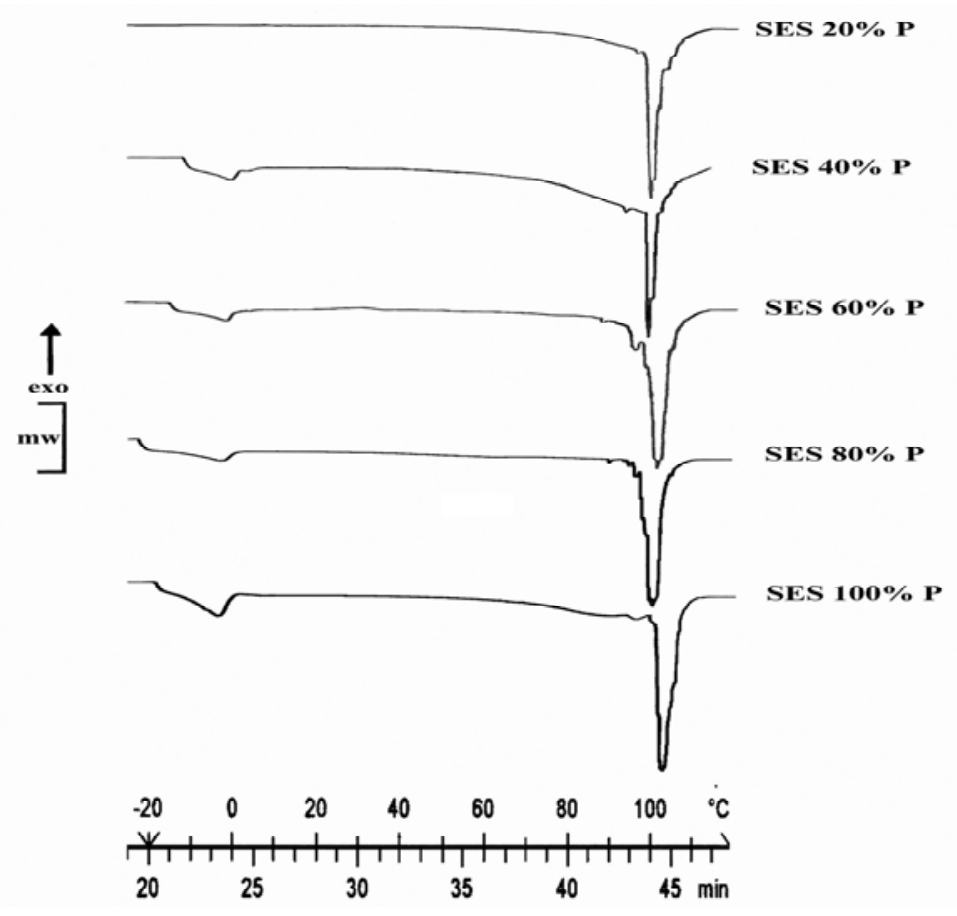

Figure 3. Thermal profiles of different SES P 

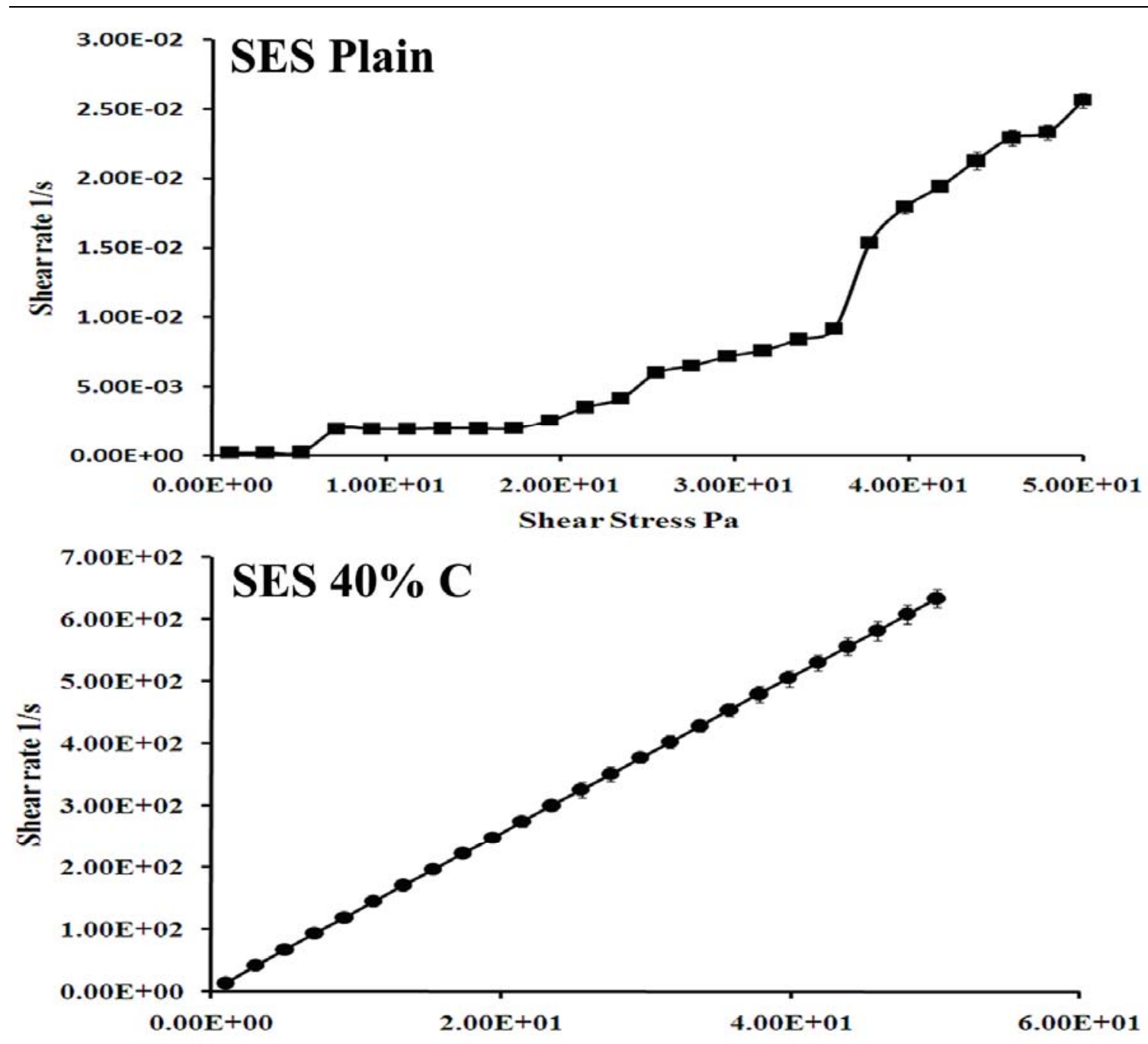

Shearstress Pa

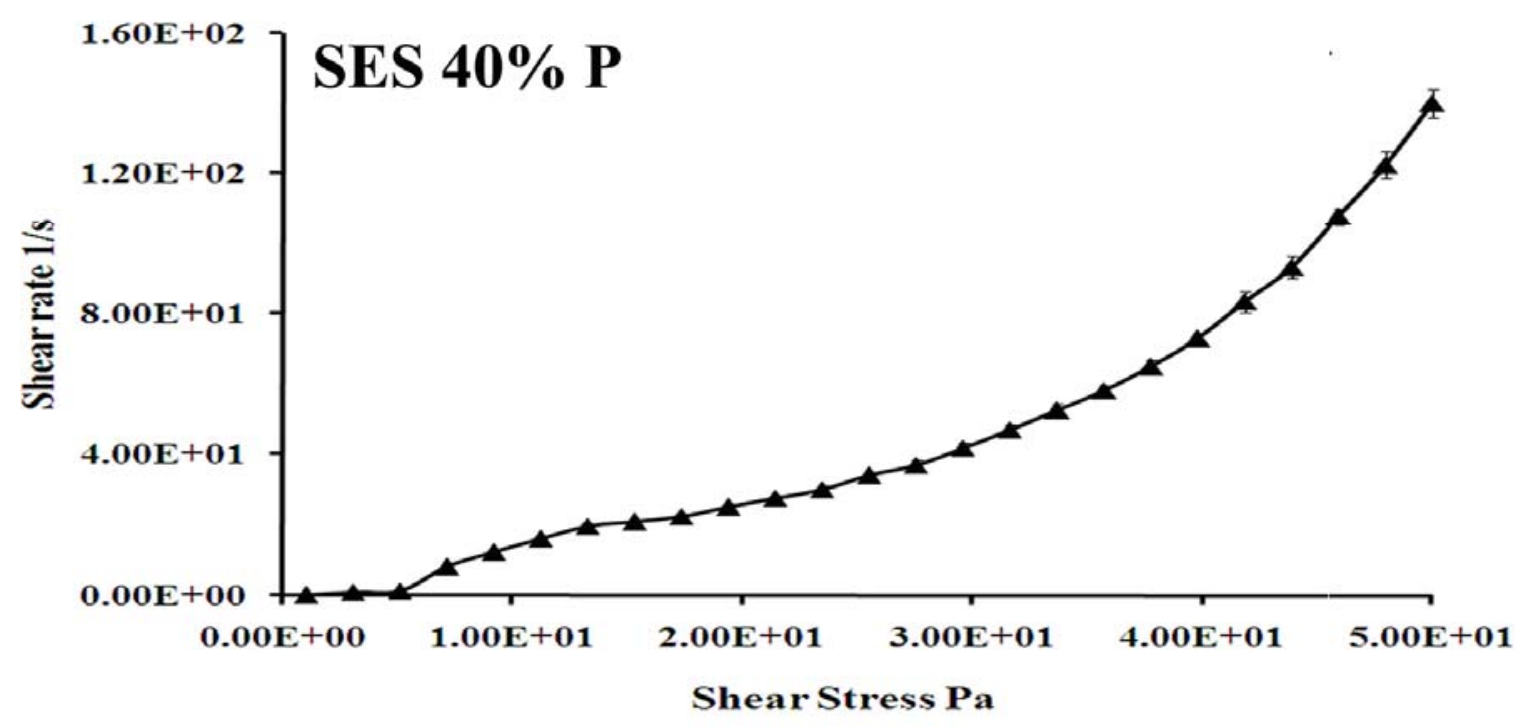

Figure 4. Rheograms of SES presenting correlation between shear stress and shear rate 
Table 3. Stress sweep results for different SES

\begin{tabular}{|c|c|c|c|c|}
\hline \multirow{2}{*}{ SES } & \multicolumn{3}{|c|}{ Stress sweep $^{\text {a }}$ (1-50 Pa) } & \multirow{2}{*}{ Observation } \\
\hline & $G^{\prime} \mathbf{P a}$ & G'P Pa & LVR Pa & \\
\hline SES Plain & $6431.00-7420.00$ & $5556.00-682.60$ & $16.0-50.0$ & $\begin{array}{l}\text { Elastic-all over } \\
\text { the stress }\end{array}$ \\
\hline SES $20 \% \mathrm{C}$ & $0.003100-0.029470$ & $0.9513-0.1244$ & $18.0-26.0$ & Viscous- all over stress \\
\hline SES $40 \% \mathrm{C}$ & $0.007676-0.005655$ & $0.1320-0.1347$ & $18.0-26.0$ & Viscous- all over stress \\
\hline SES $60 \% \mathrm{C}$ & $0.001036-0.007683$ & $0.1253-0.1251$ & $18.0-26.0$ & Viscous- all over stress \\
\hline SES $80 \% \mathrm{C}$ & $0.007360-0.008918$ & $0.1194-0.1259$ & $16.0-26.0$ & Viscous- all over stress \\
\hline SES $100 \% \mathrm{C}$ & $0.005889-0.021200$ & $0.1373-0.1310$ & $16.0-30.0$ & Viscous- all over stress \\
\hline SES $20 \% \mathrm{P}$ & $70.0700-0.0520$ & $17.620-1.160$ & $1.00-3.68$ & Elastic within LVR \\
\hline SES $40 \% \mathrm{P}$ & $83.8700-0.0098$ & $31.270-0.373$ & $1.00-2.26$ & Elastic within LVR \\
\hline SES $60 \% \mathrm{P}$ & $6.9430-0.0234$ & $5.900-0.134$ & $1.00-1.92$ & Elastic within LVR \\
\hline SES $80 \% \mathrm{P}$ & $7.6640-0.0162$ & $4.729-0.508$ & $1.00-1.63$ & Elastic within LVR \\
\hline SES $100 \% \mathrm{P}$ & $1.4615-0.0185$ & $0.944-0.588$ & $1.00-1.63$ & Elastic within LVR \\
\hline
\end{tabular}

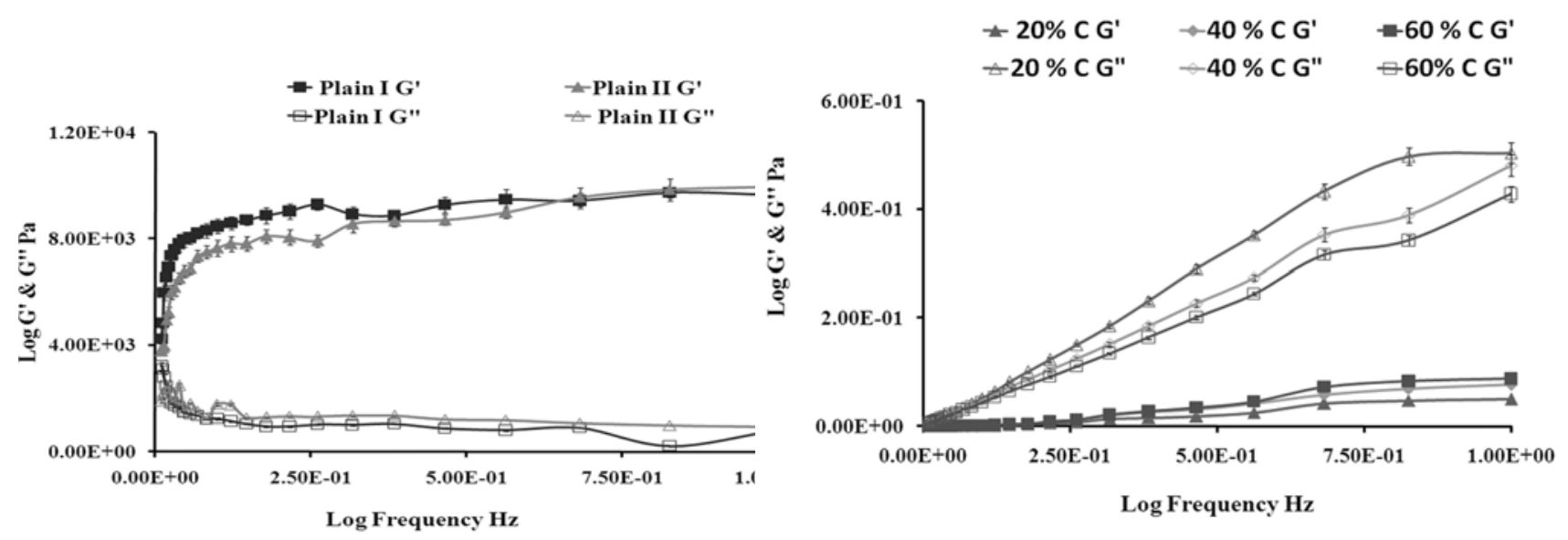

Figure 5. Frequency sweep for SES Plain I

Figure 6. Frequency sweep for studied SES C (20 Pa stress) and SES Plain II (1.5 Pa stress) 


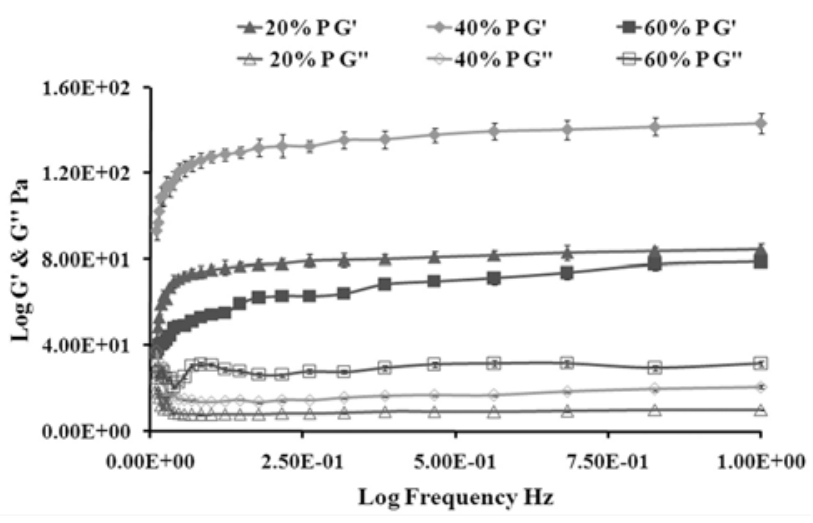

Figure 7. Frequency sweep for studied SES P

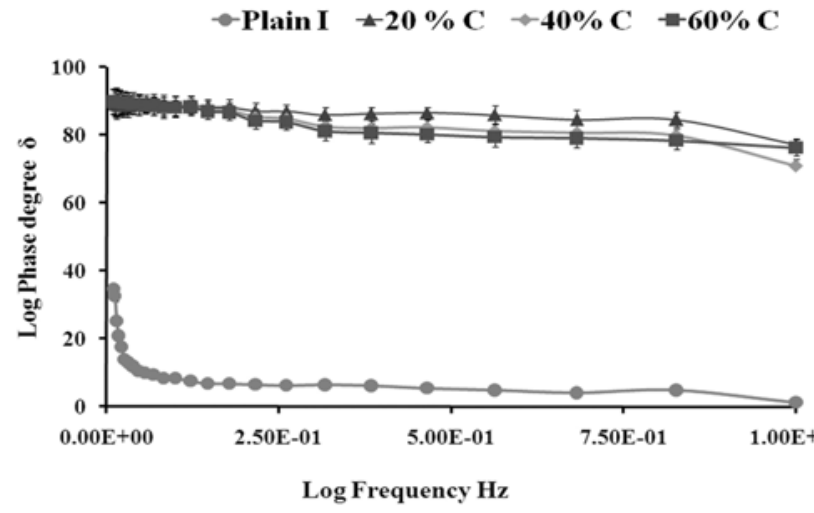

Figure 8. Effect of frequency on phase degree for SES Plain I and studied SES C

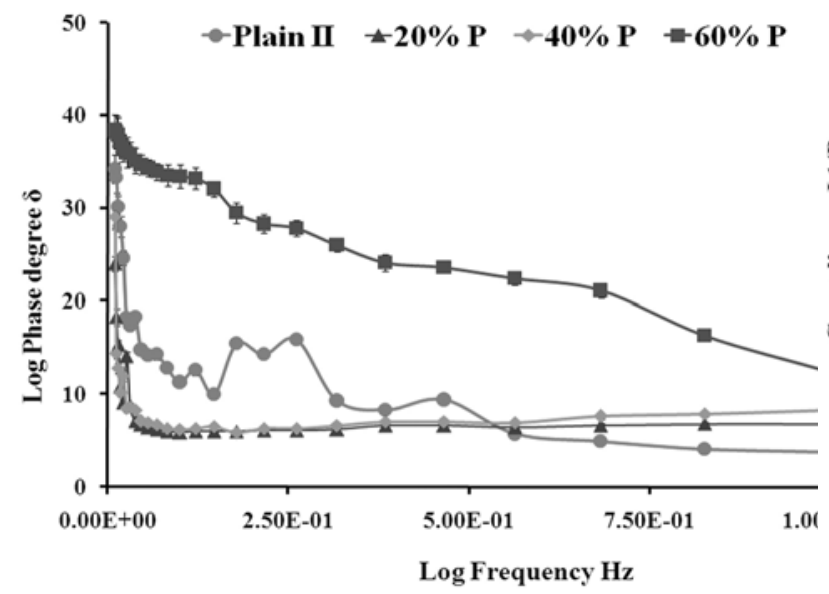

Figure 9. Effect of frequency on phase degree for SES Plain II and studied SES P

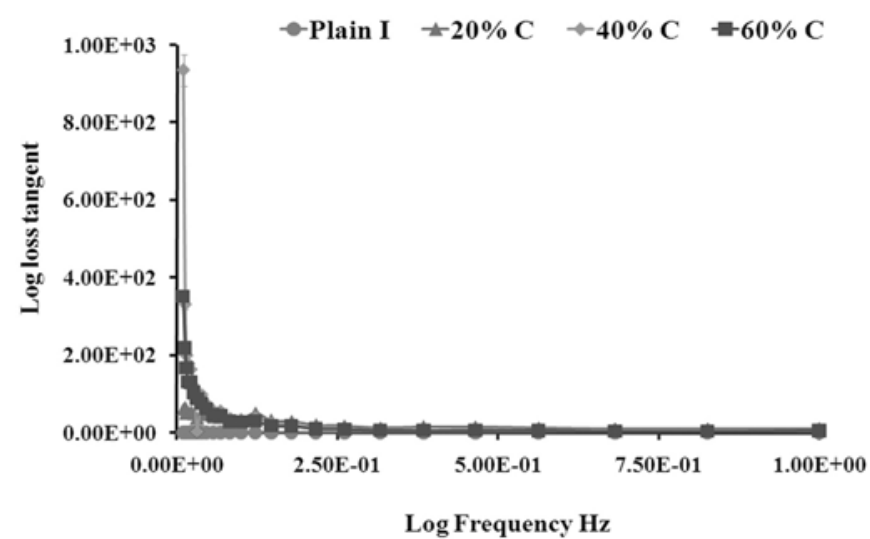

Figure 10. Effect of frequency on loss tangent for SES Plain I and studied SES C

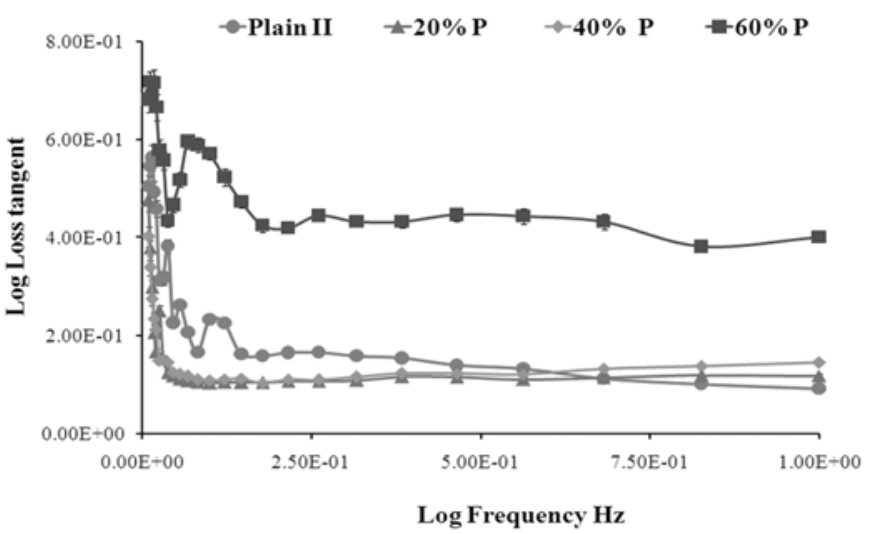

Figure 11. Effect of frequency on loss tangent for SES Plain II and studied SES P

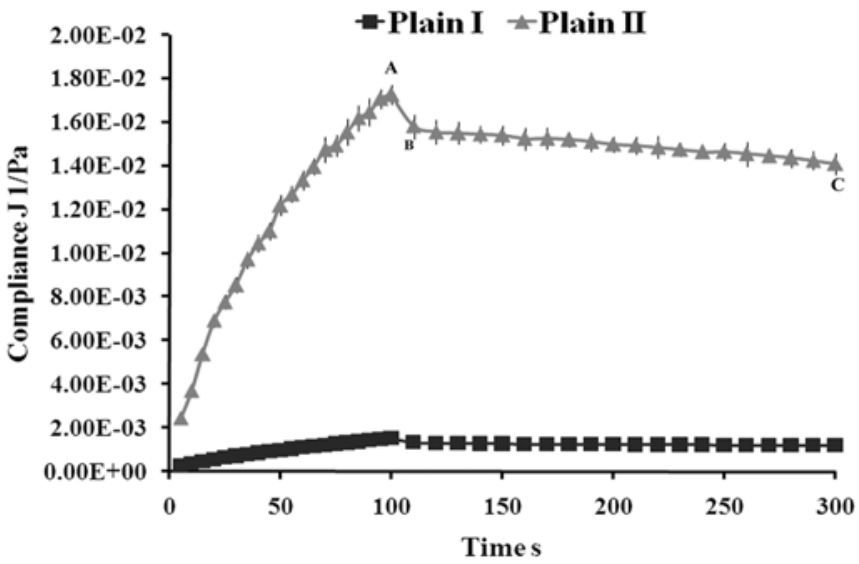

Figure 12. Creep recovery for SES Plain I (20

Pa stress) and SES Plain II (1.5 Pa stress) 


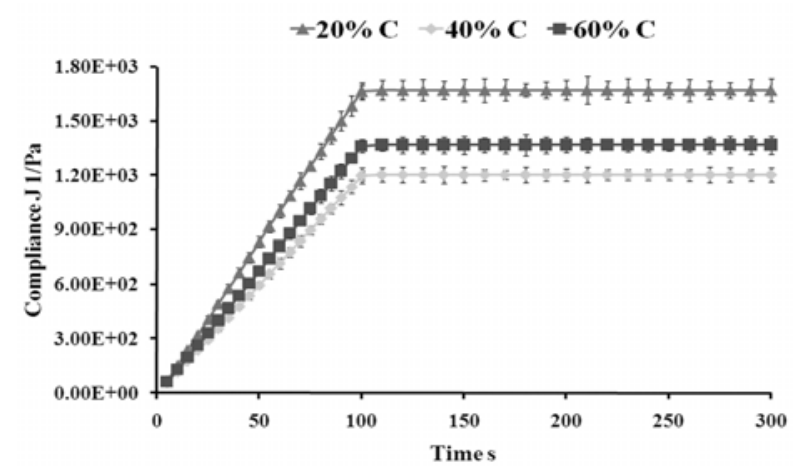

Figure 13. Creep recovery for studied SES C

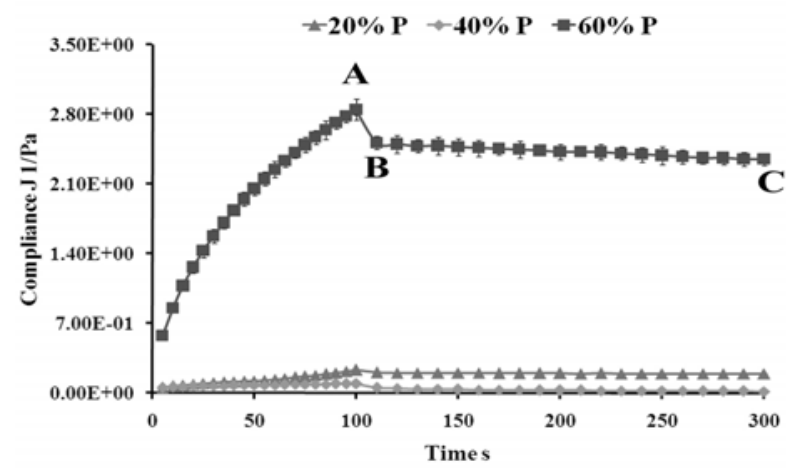

Figure 14. Creep recovery for studied SES P

\section{DISCUSSION}

The system selected for the study composed of oil (C200): surfactant (T80) and either of long hydrocarbon chain co-surfactant $(\mathrm{P})$ or medium hydrocarbon chain co-surfactant (CMCM). The system selection was based on the inability of oil; surfactant mixture to undergo spontaneous selfemulsification at selected ratio (1:1). Some mixture of oil and surfactant has been reported to self-emulsify without co-surfactant and their selfemulsification performance have been investigated previously (14). Conventionally, proportion of co-surfactant is less than that of surfactant but in this study rheological investigation of LC phase has been performed all over the range, where surfactant: co-surfactant ratio was extended up to $1: 2$ (2).

A good correlation was observed between visual observations droplet size and turbidimetric measurements. SES with lower turbidity value have 'good' emulsifying properties and have presented nano size droplets on emulsification.
For SES $80 \% \mathrm{C}$ and SES $100 \% \mathrm{C}$ with higher ratio of surfactant: co-surfactant (above 1:1), droplet size was close to one micron. The rheological properties of these SES were similar to that of SES with lower concentration of CMCM. Therefore, it can be concluded that rheological investigation was unable to reflect this minute difference in droplet size observed among different SES C. The observed difference in droplet size may be due to excess penetration of water into the bulk oil causing massive interfacial disruption and ejection of droplets into the bulk aqueous phase (2). In case of SES P, higher turbidity value and coarse droplets were observed on emulsification. Thus, self-nanoemulsification was observed for almost all SES C while selfmicroemulsification was evident in all SES P.

The extent of LC phase formation in studied SES was determined by calculating structurally bound water (SBW). The SBW determines the extent of LC phase formation; higher the SBW, higher is extent (30). From Table 4, it can be concluded that there was almost $100 \%$ LC phase formation in SES Plain and all SES C. In case of SES P, it was observed that as concentration of $\mathrm{P}$ increased, the SBW was decreased (Table 5). Decrease in the relative concentration of hydrophilic surfactant with an increase in lipophilic co-surfactant may be the underlying mechanism for such observations. Thus, lower extent of LC phase formation was evident in SES P as compared to SES C.

From rheological studies, presence of partially recoverable elastic LC phase was observed in SES Plain with only oil and surfactant. With addition of CMCM as cosurfactant to this mixture, elastic mesophase was transformed into viscous non-recoverable mesophase while elastic and partially recoverable mesophase was observed after addition of $\mathrm{P}$ as cosurfactant. Thus, rheological status of SES Plain was reversed after addition of CMCM while it was unchanged with $\mathrm{P}$ as co-surfactant. This observed difference may be attributed to the nature of molecular packaging at the interface of these two co-surfactants with different hydrocarbon chain length. It is reported that amphiphilc co-surfactant with short or medium hydrocarbon chain length has more affinity for polar head groups of the surfactant molecule due to their comparatively higher solubility and thus, cause swelling of head group of surfactant. In contrast, long chain co-surfactant cause swelling of hydrocarbon tail of surfactant due to their poor diffusion ability. This leads to an increased 
Table 4. Results for first melting transition for free water in DSC thermal profiles of SES Plain, DW and SES C

\begin{tabular}{cccccc}
\hline SES & Onset $\left({ }^{\circ} \mathbf{C}\right)$ & $\begin{array}{c}\text { Peak } \\
\left({ }^{\circ} \mathbf{C}\right)\end{array}$ & $\begin{array}{c}\text { End } \\
\left({ }^{\circ} \mathbf{C}\right)\end{array}$ & $\begin{array}{c}\text { Heat } \\
\mathbf{J} / \mathbf{g}\end{array}$ & $\begin{array}{c}\text { SBW } \\
\mathbf{\%}\end{array}$ \\
\hline SES Plain & NA & NA & NA & 0 & 100 \\
SES 20\%C & NA & NA & NA & 0 & 100 \\
SES 40\%C & -0.66 & -0.17 & 0.53 & -2.96 & 99.12 \\
SES 60\%C & NA & NA & NA & 0 & 100 \\
SES 80\%C & NA & NA & NA & 0 & 100 \\
SES 100\%C & NA & NA & NA & 0 & 100 \\
DW & -0.36 & 3.03 & 7.95 & -336.64 & NA \\
\hline
\end{tabular}

Table 5. Results for first melting transition for free water in DSC thermal profiles of SES P

\begin{tabular}{lccccc}
\hline SES & Onset $\left({ }^{\circ} \mathbf{C}\right)$ & $\begin{array}{c}\text { Peak } \\
\left({ }^{\circ} \mathbf{C}\right)\end{array}$ & $\begin{array}{c}\text { End } \\
\left({ }^{\circ} \mathbf{C}\right)\end{array}$ & $\begin{array}{c}\text { Heat } \\
\mathbf{J} / \mathbf{g}\end{array}$ & $\begin{array}{c}\text { SBW } \\
\%\end{array}$ \\
\hline SES 20\% P & NA & NA & NA & 0 & 100 \\
SES 40\% P & -17.85 & -0.24 & 4.14 & -21.76 & 93.47 \\
SES 60\% P & -17.12 & -1.60 & 4.30 & -43.25 & 87.04 \\
SES 80\% P & -18.52 & 0.66 & 4.73 & -43.84 & 86.86 \\
SES100\% P & -19.6 & -5.89 & -2.94 & -75.49 & 77.38 \\
\hline
\end{tabular}

hydrocarbon chain interaction resulting in a nonflexible interface. Therefore, in presence of longchain co-surfactant lamellar LC phase is formed or maintained at interface with greater rigidity or elasticity. On other hand, medium chain cosurfactant decreases the hydrocarbon interaction leading to transition of lamellar phase to a viscous intermediate structure (7, 8, and 27). Therefore, addition of CMCM with medium hydrocarbon chain length, transformed the elastic interface of SES Plain into a weak and viscous structure, while elastic LC phase was maintained after addition of long- hydrocarbon chain co-surfactant $P$ to SES Plain. Hence, during emulsification, the rupture pattern of elastic intermediate mesophase and a viscous mesophase will be totally different leading to the difference in size of resulting droplets.
Wakerly et al. (10) and Rang et al. (13) proposed that efficiency of self-emulsification is function of rate and extent of water penetration into formed intermediate LC phase. Therefore, observed difference in the droplet size of SES C and SES P can be correlated to the difference in viscoelastic properties of intermediate LC phase. The weak and viscous LC phase in SES C must have not presented any resistance to the strain induced deformation and hence might have ruptured easily and quickly (31). Thus, nano-size droplets are achieved from such interface disruption while elastic and strong intermediate LC phase structure in SES P must have presented comparatively higher resistance against strain induced deformation resulting in coarse droplets after emulsification. The observed outcomes are in compliance with our previous reports where 
self-emulsification performance attributed to particular ratio of oil: surfactant was evaluated by rheological analysis of intermediate LC phase (14). The observed 'moderate' self-emulsifying ability of SES P was attributed to presence of low LVR in stress sweep, slight dependence of G' and G' over applied frequency and absence of complete creep recovery percent (32).

To confirm the LC phase characterization of studied SES, $30 \% \mathrm{v} / \mathrm{v}$ hydrated SES were analyzed by small angle neutron scattering (SANS). Produced signal was observed to be very weak and therefore, analysis was non-conclusive. Pouton (2) also defined the limitation of advanced diffraction techniques like SANS to characterize the system based on vegetable oil (MCT) due to production of ill-defined spectra.

\section{CONCLUSION}

The observed difference in self-emulsification performance of these two co-surfactants was function of their influence on viscoelastic properties of LC phase, which in turn depend on co-surfactant hydrocarbon chain length. Thus, study has established the correlation between hydrocarbon chain length of co-surfactant and their self-emulsification ability. It was noted that for efficient self-emulsification, intermediate LC phase must be weak and viscous and medium chain co-surfactant have ability to yield such LC phase structure especially when a medium chain oil and hydrophilic surfactant are used for preparation of SES. Thus, significance of viscoelastic properties of intermediate LC phase was re-insisted in this study.

\section{ACKNOWLEDGMENT}

Authors are thankful to Abitec Corporation, USA for gift sample of Captex ${ }^{\circledR}$ 200P and Capmul ${ }^{\circledR}$ MCM. Authors are also grateful to Gattefosse, France for gift sample of Peceol ${ }^{\circledR}$. Shailesh V. Biradar and Ravindra S. Dhumal are thankful to Council of Industrial and Scientific Research, New Delhi, India for providing financial assistance in form of Senior Research Fellowship. Authors wish to express gratitude towards Dr. P. U. Sastry from Solid State Physics Department, Bhabha Atomic Research Center, Mumbai, India for extending small angle and neutron XRD instrument facilities for analysis of our samples.

\section{REFERENCES}

[1]. Pouton CW. Effects of the inclusion of a model drug on the performance of selfemulsifying formulations. J Pharm Pharmacol, 37: 1P, 1985.

[2]. Pouton CW. Formulation of self-emulsifying drug delivery systems. Adv Drug Del Rev, 25: 47-58, 1997.

[3]. Pouton CW. Lipid formulations for oral administration of drugs: non-emulsifying, self-emulsifying and 'self-microemulsifying' drug delivery systems. Eur J Pharm Sci, 11: S93-S98, 2000.

[4]. Gershanik T, Bonita S. Self-dispersion lipid formulations for improving oral absorption of lipophilic drugs. Eur J Pharm Biopharm, 50: 179-188, 2000.

[5]. Pouton CW, Porter, CJH. Formulation of lipid-based delivery systems for oral administration: Materials, methods and strategies. Adv Drug Del Rev, 60: 625-637, 2008.

[6]. Reiss H. Entropy-induced dispersion of bulk liquids. J Colloids Interface Sci, 53: 61-70, 1975.

[7]. Eccleston, GM., Microemulsions, in Swarbrick S: Boylan JC (eds.), Encyclopedia of Pharmaceutical Technology. 1st ed., Marcel Dekker, INC, New York, NY, pp. 375-421, 1992.

[8]. Leung, R.; Shah, DO., Microemulsions: an evolving technology for pharmaceutical applications, in Rosoff $\mathrm{M}$ (eds) Controlled Release of Drugs: Polymers and Aggregate Systems. VCH Publishers, INC., New York, NY, pp. 185-215, 1989.

[9]. Groves MJ, De Galindez DA. The selfemulsifying action of mixed surfactants in oil. Acta Phar Suec, 13: 361-372, 1976.

[10]. Wakerly MG, Pouton CW, Meakin BJ, Morton FS. Self-emulsification of vegetable oil-non-ionic surfactant mixtures. ACS Symp Series, 311: 242-55, 1986.

[11]. Pouton CW, Wakerly MG, Meakin BJ. Selfemulsifying systems for oral delivery of drugs. Proc Int Symp Control Release Bioact Mater, 14: 113-114, 1987.

[12]. Craig DQM, Barker, SA, Banning, D, Booth SW. An investigation into the mechanism of self-emulsification using particle size analysis and low frequency dielectric spectroscopy. Int $\mathrm{J}$ Pharm, 114: 103-110, 1995.

[13]. Rang MJ, Miller CA. Spontaneous emulsification of oils containing hydrocarbon, non-ionic surfactant, and oleyl alcohol. J Colloids Interface Sci, 209: 179-92, 1999.

[14]. Biradar SV, Dhumal RS, Paradkar AR. Rheological investigation of self- 
emulsification process. J Pharm Pharm Sci, 12: 17-31, 2009.

[15]. Collaborative International Pesticide Analytical Council (CIPAC), Handbook1, WHO, 1973.

[16]. Pouton CW. Self-emulsifying drug delivery systems: assessment of the efficiency of emulsification. Int J Pharm, 27: 335-348, 1985.

[17]. Taha EI, Al-Saidan S, Samy AM, Khan MA. Preparation and in vitro characterization of self-nanoemulsified drug delivery system (SNEDDS) of all-trans-retinol acetate. Int J Pharm, 285: 109-119, 2004.

[18]. Rosevear FB. The microscopy of the liquid crystalline neat and middle phases of soaps and synthetic detergents. J Am Oil Chem Soc, 31: 628-639, 1954.

[19]. Ivanova R, Lindman B, Alexandris P. Effect of pharmaceutically acceptable glycols on the stability of the liquid crystalline gels formed by poloxamer 407 in water. J Colloid Interface Sci, 252: 226-235, 2002.

[20]. Lindblom G, Rilfors L. Cubic phases and isotropic structures formed by membrane lipids- possible biological relevance. Biochim Biophys Acta, 988: 221-256, 1989.

[21]. Rodrýguez-Abreu C, Garcýa-Roman M, Kunieda H. Rheology and dynamics of micellar cubic phases and related emulsions. Langmuir, 20: 5235-5240, 2004.

[22]. Efrat R, Aserin A, Kesselman E, Danino D, Wachtel E, Garti N. Liquid micellar discontinuous cubic mesophase from ternary monoolein/ethanol/water mixtures. Colloid Surface A, 299: 133-145, 2007.

[23]. Garti N, Anserin A, Ezrahi S, Tiunova I, Berkovic B. Water behavior in nonionic surfactant systems I: subzero temperature behavior of water in nonionic microemulsions studied by DSC. J Colloid Interface Sci, 178: 60-68, 1996.
[24]. Schulz PC, Puig JE, Barreiro G, Torres LA. Thermal transitions in surfactant-based lyotropic liquid crystals. Thermochi Acta, 231: 239-256, 1994.

[25]. Antona PD, Parker WO, Zanirato MC, Esposito E, Nastruzzi C. Rheologic and NMR characterization of monoglyceride-based formulations. J Biomed Mater Res, 52: 40$52,2000$.

[26]. Lippacher A, Muller RH, Mader K. Liquid and semisolid $\mathrm{SLN}^{\mathrm{TM}}$ dispersion for topical application: rheological characterization. Eur J Pharm Biopharm, 58: 561-567, 2004.

[27]. Korhonen M. Rheological properties of pharmaceutical creams containing sorbitan fatty acid ester surfactants. Academic Dissertation, University of Helsinki, Finland, 2004

[28]. Wesis J, McClements DJ. Influence of ostwald ripening on rheology of oil-in-water emulsions containing electrostatically stabilized droplets. Langmuir, 16: 2145-2150, 2000

[29]. Tadros T. Application of rheology for assessment and prediction of the long-term physical stability of emulsions. Adv Colloid Interface, 109: 227-258, 2007.

[30]. Santos OD, Rocha-Filho PA. Influence of surfactant on the thermal behavior of marigold oil emulsion with liquid crystal phases. Drug Dev Ind Pharm, 33: 543-549, 2007.

[31]. Dabros T, Yeung A, Masliyah J, Czarnecki J. Emulsification through area contraction. J Colloids Interface Sci, 210: 222-224, 1999.

[32]. Varma, SR, Christy MW. Rheology of microcrystalline cellulose and sodiumcarboxymethyl cellulose hydrogels using a controlled stress rheometer: Part II. Int J Pharm, 292: 63-73, 2005. 